\title{
El desarrollo del lenguaje evaluativo en narraciones de niños mexicanos de 3 a 12 años
}

\author{
Evaluative language development in narratives of Mexican \\ children from 3 to 12 years
}

\author{
Silvia Romero Contreras ${ }^{1}$ \\ Gloria Elena Gómez Martínez ${ }^{2}$ \\ Universidad Autónoma de San Luis Potosí, México
}

\begin{abstract}
Resumen. Se reporta el desarrollo de la evaluación en narraciones orales de 40 niños mexicanos de 3 a 12 años. Se obtuvieron siete narraciones por sujeto a partir de láminas seleccionadas de libros sin texto. Se definió la evaluación narrativa como la expresión de la postura del narrador a partir de comentarios sobre los sucesos y los personajes, y de la expresión de las emociones de los personajes de la historia. Se construyeron categorías evaluativas tomando en cuenta estudios sobre evaluación en la narración y los propios datos. Los resultados muestran que la cantidad de evaluaciones ofrecidas y su nivel de elaboración discursiva varían en el rango de edad estudiado.
\end{abstract}

Palabras clave: narración oral, evaluación, narración infantil, desarrollo del lenguaje, español.

Abstract. This study reports on the development of evaluation in elicited oral narratives from 40 Mexican children aged three to twelve years. Each participant produced seven narratives. A series of illustrations from wordless picture books was used as elicitation material. We defined evaluation as the expression of the position of the narrator through comments about the events and characters, and the emotions of the characters in the story. The analysis included the construction of categories taking into account both the literature on evaluation in narratives, and the data itself. Results show that quantity of evaluative expressions and their level of discourse elaboration vary across ages.

Key words: Oral narrative, evaluation, child narratives, language development, spanish.

\footnotetext{
${ }^{1}$ Silvia Romero Contreras, Universidad Autónoma de San Luis Potosí. E-mail: romerosil@gmail.com

${ }^{2}$ Gloria Elena Gómez Martínez, Universidad Autónoma de San Luis Potosí. E-mail: relena2125@gmail.com

Dirección postal: Facultad de Psicología. UASLP Campus Oriente. Carretera Central Km. 424.5. San Luis Potosí, SLP. México 78494.
} 


\section{Introducción}

La narración es una forma de discurso que posee características y estructura particulares. La prolija producción de investigación sobre discurso narrativo ha dado origen a múltiples enfoques para su estudio y a múltiples definiciones. En esta investigación se define la narración como un discurso que cumple con al menos las siguientes dos características (Hess, 2010): tiene una secuencia temporal que se manifiesta gramaticalmente (generalmente en tiempo pretérito) y hay una ruptura de la canonicidad, también conocida como complicación (Labov \& Waletzky, 1967) u obstáculo (Stein \& Albro, 1997).

Un aspecto central de la narración, es la postura del narrador, es decir, la manera en que el narrador percibe y comunica el hecho narrado. Esta característica, denominada función expresiva (Labov \& Waletzky, 1967), se manifiesta mediante diversos mecanismos de evaluación que si bien son subjetivos, reflejan las normas y expectativas de la cultura a la que pertenece el narrador (Fernández \& Melzi, 2008).

El estudio del desarrollo del lenguaje evaluativo en la narración ha mostrado que existen diferencias transculturales. La mayor parte de las investigaciones en esta área se han realizado con poblaciones anglosajonas y asiáticas; los estudios con poblaciones hispanohablantes siguen siendo escasos (Fernández \& Melzi, 2008).

El propósito de este trabajo es describir el desarrollo del lenguaje evaluativo de niños de 3 a 12 años a través de la identificación y caracterización del tipo de emociones de los personajes de la historia, así como los comentarios que expresan en narraciones producidas a partir de láminas.
La narración es una actividad social que vincula a las personas, permitiendo que compartan experiencias. Labov y Waletzky (1967) plantean que las narraciones incluyen una postura que es el motivo por el cual se narra, la cual se expresa a través de las evaluaciones que el narrador realiza durante su relato. Las cláusulas evaluativas suelen suspender la acción antes de un evento crítico y en ese momento el narrador suele expresar sus sentimientos: citándose a sí mismo o citando una tercera persona. Estas evaluaciones pueden reflejar la interpretación que el narrador hace de las emociones de los personajes (Aldrich, Tenenbaum, Brooks, Harrison, \& Sines, 2011; Bamberg \& Reilly, 1996; Jiménez Van Der Biest, 2006; Levey, 2003; Shiro, 1998), o su postura ante el hecho narrado, por medio de la expresión de sus comentarios, creencias o percepciones (Jiménez Van Der Biest, 2006; Shiro, 1998).

De acuerdo con Shiro (2008), el tipo de narración, personal o de ficción, influye en las evaluaciones que se expresan; las interpretaciones sobre emociones, que se expresan en tercera persona, son más frecuentes en las narraciones de ficción; mientras que la postura del narrador, que se expresa en primera persona, es más frecuente en las narraciones personales.

La presencia de lenguaje evaluativo varía en función de edad y la situación comunicativa. Fernández y Melzi (2008) contrastaron las referencias a estados internos de niños y niñas de 3 y 5 años en interacción con su madre en dos situaciones: conversaciones sobre experiencias personales y lectura compartida del libro "Frog, where are you?". Al contrastar ambas situaciones, encontraron significativamente más estados internos en las narraciones a partir del libro que en las conversaciones, sin importar la edad o el género. Al analizar las situaciones por separado, 
no encontraron diferencias significativas por edad o género en las referencias a estados internos en caso del libro, pero sí en el caso del libro, pero sí, en el de la conversación, a favor de las niñas y el grupo de edad más alto.

En una revisión de dos estudios con narraciones producidas por niños y adultos a partir del libro de "Frog, where are you?", Bamberg y Reilly (1996) encontraron que al inicio del desarrollo narrativo la expresión de emociones de los personajes parece obedecer a una situación más bien estilística; es decir, los niños entienden que, como sus padres hablan de emociones en sus narraciones, éste es un elemento que deben incluir para hacer una buena narración. Los niños en esta etapa no analizan los sentimientos de los personajes como parte de la historia, por lo que la expresión de emociones puede no estar incluida en la lógica de la historia (se puede estar hablando sobre un niño dormido y mencionar: "y la rana estaba feliz" y regresar al niño y mencionar que estaba con su perro).

Entre los seis y los ocho años la expresión de emociones se utiliza en forma local dentro de las cláusulas narrativas; es decir, los niños empiezan a identificar emociones de los personajes, aunque en ocasiones solo se enfocan en emociones que ellos mismos han experimentado, por lo que falta información relevante de la historia (se mencionará que "el niño se preocupó cuando no vio a su rana" solamente si el narrador ha tenido la experiencia de preocuparse por algo que ha perdido; a pesar de ser un elemento fundamental de esta narración); y hacia los 10 años, las emociones de los personajes se expresan más frecuentemente, tal como se aprecian en la historia, por lo que se articula la expresión de emociones con la coherencia del relato y se le proporciona mayor coherencia a la historia y un sentido más global a la evaluación (se mencionan los momentos en que el niño estaba preocupado, cuando se asustó y al final cuando se puso feliz al encontrar a su rana).

Esta progresión en la expresión de emociones es congruente con la capacidad que poseen los niños para interpretar las imágenes de los cuentos. Aldrich et al. (2011) investigaron en niños de cinco a ocho años la relación entre comprensión de emociones (evaluada mediante el uso de una prueba estandarizada) y la expresión de emociones en narraciones obtenidas a partir del libro "One frog too many" (Mayer, 1975). Esta historia gira alrededor de la envidia, y la trama consiste en que una rana se pone celosa cuando su dueño recibe otra ranita de regalo. Las puntuaciones de los niños en la prueba de comprensión de emociones mostraron un incremento hacia los siete años lo que se relacionó con una mayor habilidad para expresar la idea de envidia en la historia y con la capacidad de los niños para identificar al personaje "rival" mientras hacían su narración (Aldrich et al., 2011).

La expresión de emociones muestra un desarrollo similar cuando se utilizan otras estrategias de producción, como la narración personal, contar películas o producir narraciones escritas. En un análisis de narraciones escritas producidas por alumnos de $2^{\circ}$ y $5^{\circ}$ grados de educación básica a partir de láminas, se observó que la presencia de menciones directas a los estados emocionales de los personajes aumenta en forma sensible con la edad; es decir las emociones, como estar alegre o enojado y los sentimientos, como expresar que dos hermanos se querían (Jiménez Van Der Biest, 2006).

En cuanto a la expresión de comentarios, creencias y opiniones por parte del narrador, 
se ha encontrado que su uso es escaso hasta alrededor de los seis años, coincidente con la fase intermedia del desarrollo de la emotividad narrativa según Bamberg y Reilly (1996). Hacia los ocho años el narrador se vuelve más presente, con lo que contribuye a la organización y construcción global de la historia (Aldrich, et al., 2011; Bamberg \& Reilly, 1996). Otros estudios analizan la perspectiva que asume el niño como narrador a la hora de relatar.

De acuerdo con Shiro (1998), la tendencia evolutiva es primero expresar un reporte directo de las emociones (tercera persona: "está enojada") y posteriormente realizarlo de manera personal (primera persona: "creo que está enojada"). En el análisis por tipo de narración (personal o de ficción) encontró que las evaluaciones desde la perspectiva del personaje (expresadas en $3^{\mathrm{a}}$ persona) son más frecuentes en las narraciones de ficción y las evaluaciones desde la perspectiva del narrador (expresadas en $1^{\mathrm{a}}$ persona) son más frecuentes en las narraciones personales, como lo había anticipado (Shiro, 2008). No obstante, un análisis realizado por Shiro (2003, 2008) contrastando las narraciones por nivel socioeconómico, mostró que solo los niños de nivel alto (escuela privada) muestran diferencias significativas relacionadas con el grado escolar $\left(1^{\circ}\right.$ vs. $4^{\circ}$ grado) en la calidad de su lenguaje evaluativo.

En el trabajo de Jiménez Van Der Biest (2006) con narraciones escritas, también se observó un incremento en las evaluaciones en tercera persona entre $2^{\circ}$ y $5^{\circ}$ grados, aunque en este estudio se concluye que la habilidad de expresar emociones en primera persona no se encontró desarrollada de manera evidente en estos grados escolares.

En términos generales los diversos estudios coinciden en hallar una línea evolutiva en la expresión narrativa en relatos no personales, es decir, en los que el narrador no es el protagonista. Esta evolución inicia con el reporte de emociones de los personajes en forma directa. Más adelante el niño hace uso de evaluaciones personales donde el narrador expresa una postura ante el hecho, enfatizando emociones con las que se identifica y presentándolas desde una perspectiva más personal.

En este trabajo interesa, conocer cómo progresa la evaluación narrativa en una población de niñas y niños hispanohablantes de 3 a 12 años de nivel socioeconómico bajo, en relatos obtenidos con series de láminas de los libros de la colección de "Frog". Las evaluaciones obtenidas en estas narraciones de ficción se analizaron considerando la presencia de emociones y comentarios y sus subtipos. En el caso de las emociones, se distinguió entre emociones de acción y emociones de estado; en el caso de los comentarios, se diferenció entre generales (regularmente expresados en $3^{\mathrm{a}}$ persona) y personales (expresados en $1^{a}$ persona).

Además, se clasificaron las evaluaciones en tres niveles de elaboración según su complejidad. Las hipótesis en relación con estas variables fueron las siguientes: 1) por tratarse de rasgos más evidentes, las acciones (EM_Acción) son más tempranas y frecuentes que los estados (EM_Estado), los cuales requieren de una interpretación (Aldrich, et al., 2011; Jiménez Van Der Biest, 2006). 2) Los comentarios generales (CO_General) son más tempranos y frecuentes que los comentarios personales (CO_Personal) pues los segundos implican un posicionamiento del narrador (Shiro, 1998, 2008). 3) La elaboración (E1, E2 y E3) se haría más compleja con la edad (Bamberg \& Reilly, 1996). 


\section{Método}

En este trabajo se utilizaron datos de un proyecto mayor en el que se realizó la construcción y piloteo Prueba de Evaluación Narrativa (PEN) (Gómez Martínez, 2012). En dicha prueba, una de las categorías es la de evaluación, la cual se recuperó y reanalizó para dar cuenta del desarrollo de la evaluación narrativa en forma más detallada.

\section{Participantes}

En el estudio se incluyen las narraciones de 40 participantes (20 niñas y 20 niños) en un rango de edad de 3 a 12 años que asistían a un jardín infantil $(n=12)$ y una primaria $(n=28)$. Ambas escuelas son públicas y están ubicadas en una zona urbano-marginal.

La estratificación social por tipo de escuela y lugar de residencia en México es muy marcada (Instituto Nacional para la Evaluación de la Educación, 2007). Generalmente, las familias con cierto poder adquisitivo hacen lo posible por enviar a sus hijos a escuelas privadas y por vivir en zonas urbanas, en tanto que, las familias con escasos recursos llevan a sus hijos a la escuela pública y viven en zonas urbano marginales o rurales. Dado que ambas escuelas son públicas y se sitúan en zonas urbano-marginales, se puede decir que los participantes pertenecen a un estrato socioeconómico bajo.

Los participantes fueron seleccionados aleatoriamente dentro de las escuelas, las cuales fueron elegidas por ofrecer facilidades de acceso. Algunos criterios de exclusión fueron: la presencia de problemas de lenguaje y/o aprendizaje (reportados por los docentes de las escuelas) y la ausencia del niño/a el día de la evaluación. Los derechos de los participantes fueron protegidos mediante un consentimiento informado firmado por los padres, en el que se explicaron los objetivos del estudio, las pruebas que se aplicarían y que el uso de los datos sería anónimo y exclusivamente para efectos de investigación.

\section{Instrumentos}

Para obtener las narraciones se emplearon los estímulos construidos para la Prueba de Evaluación Narrativa (PEN) con los cuales se obtuvieron siete relatos por sujeto. Tanto el material de la prueba como su administración están descritos en el manual de aplicación de la prueba (Gómez Martínez, 2012). El registro de las narraciones fue preciso y objetivo al grabarlo. Se utilizó una grabadora de audio Olympus VN4100 PC para registrar las producciones de los niños y niñas.

Los reactivos de la PEN consisten en presentar al niño una selección de láminas de los libros de los autores Mercer y Mayer: "Frog, where are you?" (1969), "Frog on his own" (1973), " $A$ boy, a dog and a frog" (1967), "One frog too many" (1975) y “ $A$ boy, a dog, a frog and friend” (1971). Cada conjunto de láminas cuenta una historia completa. En la tabla 1, se describe cómo están conformados los reactivos. Los detalles de la construcción del instrumento y sus características psicométricas pueden consultarse en Gómez Martínez (2012).

Se trabajó con los participantes de manera individual. La estrategia de producción consistió en entregar al niño/a el conjunto de láminas que integraban el reactivo pidiéndole que las viera con atención (sin límite de tiempo) para que después platicara el cuento al examinador. Se solicitó a los participantes que fueran muy específicos al narrar su cuento, ya que el examinador no podía ver las láminas, pues había una pantalla de por medio. Al finalizar cada relato, se aclaraba que 
Tabla 1

Descripción de los Reactivos

\begin{tabular}{|c|c|c|c|}
\hline $\begin{array}{l}\text { Reactivo } \\
\text { Núm. }\end{array}$ & Libro & $\begin{array}{l}\text { Láminas del libro } \\
\text { (sucesos) }\end{array}$ & Descripción general del reactivo \\
\hline Reactivo ej. & $\begin{array}{l}\text { A boy, a dog and a } \\
\text { frog (Mayer, 1967) }\end{array}$ & $4,5,6,8(4)$ & $\begin{array}{l}\text { Un niño y su perro intentan atrapar una rana en un } \\
\text { lago. }\end{array}$ \\
\hline Reactivo 1 & $\begin{array}{l}\text { Frog on his own } \\
\text { (Mayer, 1973) }\end{array}$ & $9,10,12,13(4)$ & $\begin{array}{l}\text { Una pareja está de día de campo y una rana los } \\
\text { interrumpe. }\end{array}$ \\
\hline Reactivo 2 & $\begin{array}{l}\text { Frog on his own } \\
\text { (Mayer, 1973) }\end{array}$ & $14-17(4)$ & $\begin{array}{l}\text { Un niño está jugando en el lago de un parque y } \\
\text { una rana brinca a su barquito de juguete. }\end{array}$ \\
\hline Reactivo 3 & $\begin{array}{l}\text { Frog on his own } \\
\text { (Mayer, 1973) }\end{array}$ & $18,19,21-24(6)$ & $\begin{array}{l}\text { Una señora está en un parque con su bebé y una } \\
\text { rana intenta tomarse el biberón del bebé. }\end{array}$ \\
\hline Reactivo 4 & $\begin{array}{l}\text { Frog where are you? } \\
\text { (Mayer, 1969) }\end{array}$ & $2,3,8,21,23,24(6)$ & $\begin{array}{l}\text { Una rana se escapa del frasco donde la tiene un } \\
\text { niño y éste la busca en el bosque. }\end{array}$ \\
\hline Reactivo 5 & $\begin{array}{l}\text { A boy, a dog, a frog } \\
\text { and friend (Mayer \& } \\
\text { Mayer, 1971) }\end{array}$ & $2,4,5,7(4)$ & $\begin{array}{l}\text { Un niño está con su perro y su rana pescando en } \\
\text { un lago y algo jala su caña. }\end{array}$ \\
\hline Reactivo 6 & $\begin{array}{l}\text { Frog on his own } \\
\text { (Mayer, 1973) }\end{array}$ & $3,5,6,8(4)$ & $\begin{array}{l}\text { Una rana se queda sola en un parque y se come un } \\
\text { insecto, descubriendo que no es lo que creía. }\end{array}$ \\
\hline Reactivo 7 & $\begin{array}{l}\text { One frog too many } \\
\text { (Mayer \& Mayer, } \\
\text { 1975) }\end{array}$ & $1,3,4,6,7(5)$ & $\begin{array}{l}\text { Un niño recibe de regalo una ranita, por lo que su } \\
\text { perro y su tortuga se ponen felices, pero su rana se } \\
\text { pone celosa. }\end{array}$ \\
\hline
\end{tabular}

verían enseguida una nueva aventura de la rana, personaje que siempre aparecía en las láminas. Todas las producciones se grabaron en audio.

\section{Estrategias de análisis}

El protocolo de la PEN incluye varias categorías. Para el presente estudio únicamente se trabajó con la categoría "Evaluación" que incluye dos indicadores: descripción de emociones de personajes y comentarios del narrador. La categoría de evaluación fue una de las más productivas en el estudio de la PEN, el cual se realizó con 100 sujetos. El indicador "emociones" mostró una correlación significativa positiva con la edad en cuatro de los siete reactivos, mientras que el indicador "comentarios" mostró una correlación significativa positiva con la edad en todos los reactivos de la prueba; esto quiere decir que los niños mayores emplean más estos indicadores que los niños menores (Gómez Martínez, 2012) .

En este trabajo, las emociones se subclasificaron en acciones (EM_Acción) y estados (EM_Estado) y los comentarios en generales (CO_General) y personales (CO_Personal). Además, se identificó el nivel de elaboración discursiva y sintáctica de las emisiones de evaluación en tres niveles (E1, E2 y E3). Los criterios de la recodificación de la categoría de evaluación se muestran en la tabla 2.

La subclasificación de emociones se realizó considerando que las acciones reflejan emociones de los personajes, cuya interpretación parece 
Tabla 2

Recodificación de la categoria "Evaluación"

\begin{tabular}{|c|c|c|}
\hline Indicador/ & Libro & Descripción general del reactivo \\
\hline \multicolumn{3}{|l|}{ Emociones } \\
\hline /EM_Acción & $\begin{array}{l}\text { Se describen acciones que reflejan las emociones } \\
\text { de los personajes; suelen ser más evidentes en los } \\
\text { gráficos y su interpretación parece más directa: } \\
\text { llorar, gritar, reír, espantar a, etc. }\end{array}$ & $\begin{array}{l}\text { "El niño empezó a llorar cuando vio su } \\
\text { barquito hundido" }\end{array}$ \\
\hline /EM_Estado & $\begin{array}{l}\text { Se describen estados emocionales a partir } \\
\text { de las expresiones de los personajes; éstas } \\
\text { suelen ser menos evidentes en los gráficos y } \\
\text { su interpretación parece más personal: feliz, } \\
\text { enojado, asustarse, espantarse, dar envidia; estar } \\
\text { confundido, etc. }\end{array}$ & $\begin{array}{l}\text { "El perro y la tortuga se pusieron felices } \\
\text { con la rana bebé" }\end{array}$ \\
\hline \multicolumn{3}{|l|}{ Comentarios } \\
\hline /CO_General & $\begin{array}{l}\text { El comentario se refiere a la situación o los } \\
\text { personajes, sin marcar explícitamente un } \\
\text { posicionamiento del narrador. }\end{array}$ & $\begin{array}{l}\text { "Y luego la rana salta y el gato no se da } \\
\text { cuenta y salta" }\end{array}$ \\
\hline /CO_Personal & $\begin{array}{l}\text { El comentario se refiere a la situación o los } \\
\text { personajes, marcando explícitamente un } \\
\text { posicionamiento del narrador, a través de frases } \\
\text { como: yo creo, me parece, parece, etc. }\end{array}$ & $\begin{array}{l}\text { "Yo creo que era cumpleaños del niño, } \\
\text { porque tenía una caja de regalo enfrente" }\end{array}$ \\
\hline \multicolumn{3}{|c|}{ Nivel de Elaboración } \\
\hline $\begin{array}{l}\text { /E1. Sin } \\
\text { elaboración }\end{array}$ & $\begin{array}{l}\text { Se menciona la emoción o el comentario } \\
\text { sin justificarlo o sin elaborar en las causas o } \\
\text { consecuencias. }\end{array}$ & $\begin{array}{l}\text { "La niña estaba llorando y el perro } \\
\text { persiguió a la rana" }\end{array}$ \\
\hline $\begin{array}{l}\text { /E2. Indirecta } \\
\text { o implícita }\end{array}$ & $\begin{array}{l}\text { El comentario o emoción se relaciona con lo } \\
\text { dicho antes o después por el narrador, pero el } \\
\text { oyente debe hacer la "conexión" o inferencia. En } \\
\text { general se marcan por yuxtaposición o por nexos } \\
\text { como " } y \text { " }\end{array}$ & $\begin{array}{l}\text { "La empezó a buscar en el jardín, en el } \\
\text { pasto y no la encontró" }\end{array}$ \\
\hline /E3. Explícita & $\begin{array}{l}\text { El narrador da una explicación de su comentario } \\
\text { o expresión de emoción principalmente a través } \\
\text { de nexos causales (porque, por eso) o ilativos } \\
\text { (entonces). }\end{array}$ & $\begin{array}{l}\text { "Y regañaron al sapo porque la había } \\
\text { mordido" }\end{array}$ \\
\hline
\end{tabular}

más directa porque se observa de manera clara en la lámina; en tanto que los estados reflejan emociones que pueden sentir los personajes, pero que requieren una interpretación por parte del narrador. Se hizo esta diferenciación bajo la hipótesis de que las acciones serían más tempranas que los estados, por ser más evidentes.

Al subclasificar los estados se buscó identificar el posicionamiento del narrador en la historia, ya que los comentarios generales hacen referencia 
a situaciones o actitudes de los personajes de manera descriptiva, regularmente expresados en tercera persona, mientras que los comentarios personales incluyen explícitamente una toma de posición o perspectiva al hacerse, ya sea en primera persona, o marcando explícitamente que se trata de una percepción personal (ej. me parece, parece, se ve). Considerando los hallazgos de investigaciones previas (Aldrich et al., 2011; Bamberg y Reilly, 1996; Shiro, 1998, 2008), se partió de la hipótesis de que los comentarios personales serían más tardíos que los generales.

El tercer indicador, "elaboración”, que se aplicó tanto a emociones como a comentarios, ofrece una escala de tres niveles de complejidad discursiva. Este indicador se construyó bajo la hipótesis de que la complejidad se incrementaría en función de la edad.

De esta forma, los datos de la categoría evaluación se codificaron considerando cuatro subcategorías EM_Acción, EM_Estado, CO_General, CO_Personal y tres niveles de elaboración. La codificación fue realizada por las autoras de este trabajo, quienes codificaron los datos de manera independiente y los compararon. En los casos de discrepancia se precisaron las definiciones hasta llegar a un acuerdo total, y se revisaron los datos con los nuevos criterios.

Una vez codificados los datos, se obtuvieron frecuencias por sujeto y edad, y las medias totales y por edad de los indicadores, sus respectivas subcategorías y niveles de elaboración, siempre para el total de los reactivos. Estos datos permiten describir las características de la evaluación en los 10 rangos de edad. Dado que en cada rango de edad había solo cuatro sujetos, se agruparon los datos en tres grupos de edad considerando los hallazgos en el análisis descriptivo por edad, con el fin de poder probar las hipótesis planteadas utilizando análisis de varianza ANOVA. Los tres grupos quedaron como sigue: 3 a 6 años $(n=$ 16), 7 a 9 años $(n=12), 10$ a 12 años $(n=12)$.

\section{Resultados}

Para tener una perspectiva global del fenómeno de la evaluación en las narraciones infantiles de ficción, se analizó el uso de los tipos de evaluación y su nivel de elaboración en el total de la muestra $(N=40)$.

Como se observa en la tabla 3, se obtuvo un total de 781 evaluaciones. La evaluación a través de la expresión de emociones, y dentro de éstas las emociones de estado (EM_Estado), fue más frecuente que la evaluación a través de comentarios, donde predominaron por muy amplio margen los comentarios generales (CO_General). Esto significa que en estas narraciones de ficción el discurso evaluativo se centró, por una parte, en describir los estados emocionales que los personajes reflejan a través de sus expresiones (estar feliz, enojado, etc.) más que de sus acciones (llorar, gritar, etc.), y por la

Tabla 3

Descriptivas por tipo de evaluación y subcategoría $(N=40)$

\begin{tabular}{lcccc}
\hline Tipo de Evaluación & Media & DE & Rango & Total \\
\hline Emociones & 11.3 & 4.8 & $4-24$ & 452 \\
EM_Acción & 2.53 & 1.41 & $0-6$ & 101 \\
/EM_Estado & 8.78 & 3.9 & $2-20$ & 351 \\
Comentarios & 8.23 & 6.13 & $0-24$ & 329 \\
$\quad$ /CO_General & 7.83 & 5.6 & $0-24$ & 313 \\
/CO_Personal & 0.4 & 1.37 & $0-7$ & 16 \\
Nivel de Elaboración & & & & \\
/E1. Sin elaboración & 6.68 & 4.48 & $0-19$ & 267 \\
/E2. Indirecta o & 7.95 & 5.66 & $0-22$ & 318 \\
implícita & & & & \\
/E3. Explícita & 4.9 & 5.78 & $0-30$ & 196 \\
Total Evaluaciones & 19.53 & 10.03 & $5-43$ & 781 \\
\hline
\end{tabular}


otra, en comentar lo que sucede en la historia sin incluir un posicionamiento explícito del narrador (yo creo...). Predominó, además, el discurso evaluativo de elaboración simple e intermedia (E1. Sin elaboración y E2. Indirecta o implícita).

Se obtuvieron las medias de respuesta por indicador y por edad. La figura 1 presenta los resultados de los dos tipos de evaluación (Emociones y Comentarios) por edad. Se observó una tendencia ascendente en ambos indicadores. Sin embargo, las emociones son más frecuentes desde el inicio y se disparan a los 10 años, en tanto que los comentarios van ascendiendo gradualmente hasta los nueve años y posteriormenteseincrementandemaneranotable.

Las respuestas de los participantes seleccionados $(N=40)$ se recodificaron aplicando las subcategorías descritas en la tabla 2 , y se calcularon las medias por edad en el total de los relatos. En la figura 2, se muestran las tendencias encontradas. Como puede observarse, las subcategorías de emociones de estado (EM_Estado) y comentarios generales
(CO_General) fueron las más productivas, mostrando tendencias ascendentes en relación con la edad. Por su parte, las emociones de acción (EM_Acción) y los comentarios personales (CO_Personal) ocurren poco y con incrementos muy menores a lo largo de todas las edades.

Para comprender mejor cómo evoluciona la expresión de la evaluación, las producciones se analizaron considerando su elaboración discursiva, tomando en cuenta tres niveles (ver tabla 2). En la figura 3, se presenta la distribución por nivel de elaboración. Como puede observarse, predominan las evaluaciones sin elaboración (E1) hasta los seis años; a partir de los siete años se observa un incremento sensible de la elaboración indirecta o implícita (E2) que posibilita al oyente inferir, con base en lo dicho, cómo se relaciona la evaluación con el resto del relato. La evaluación con elaboración explícita (E3) ocurre escasamente entre los tres y los ocho años, a partir de los nueve años, se hace más frecuente pero no de manera consistente en todas las edades.

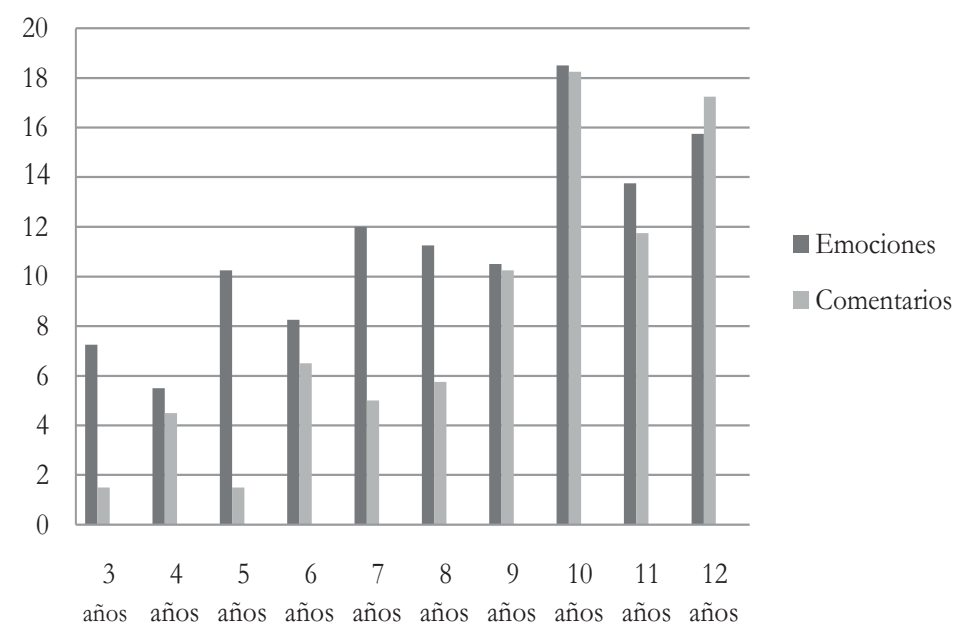

Figura 1. Emociones y Comentarios. Medias por edad en el total de los relatos $(N=40)$. 


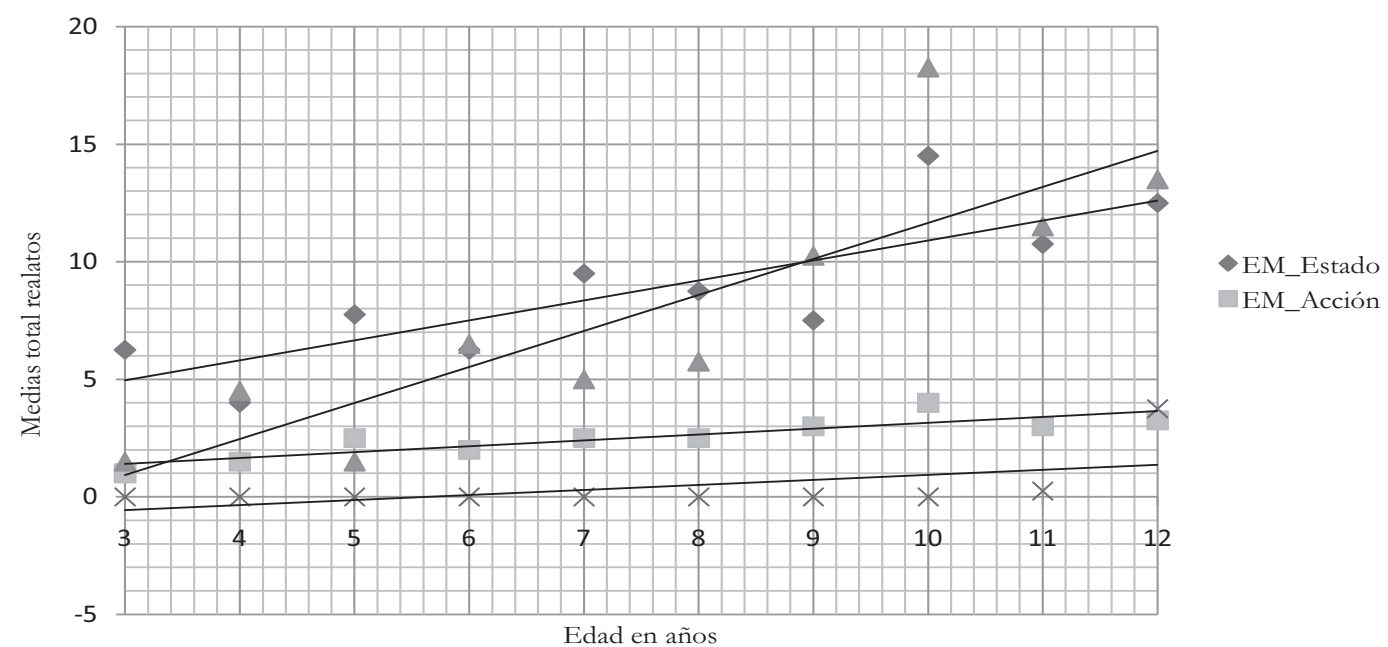

Figura 2. Tendencias de las subcategorías de Emociones y Comentarios. Medias por edad en el total de los reltatos $(N=40)$.

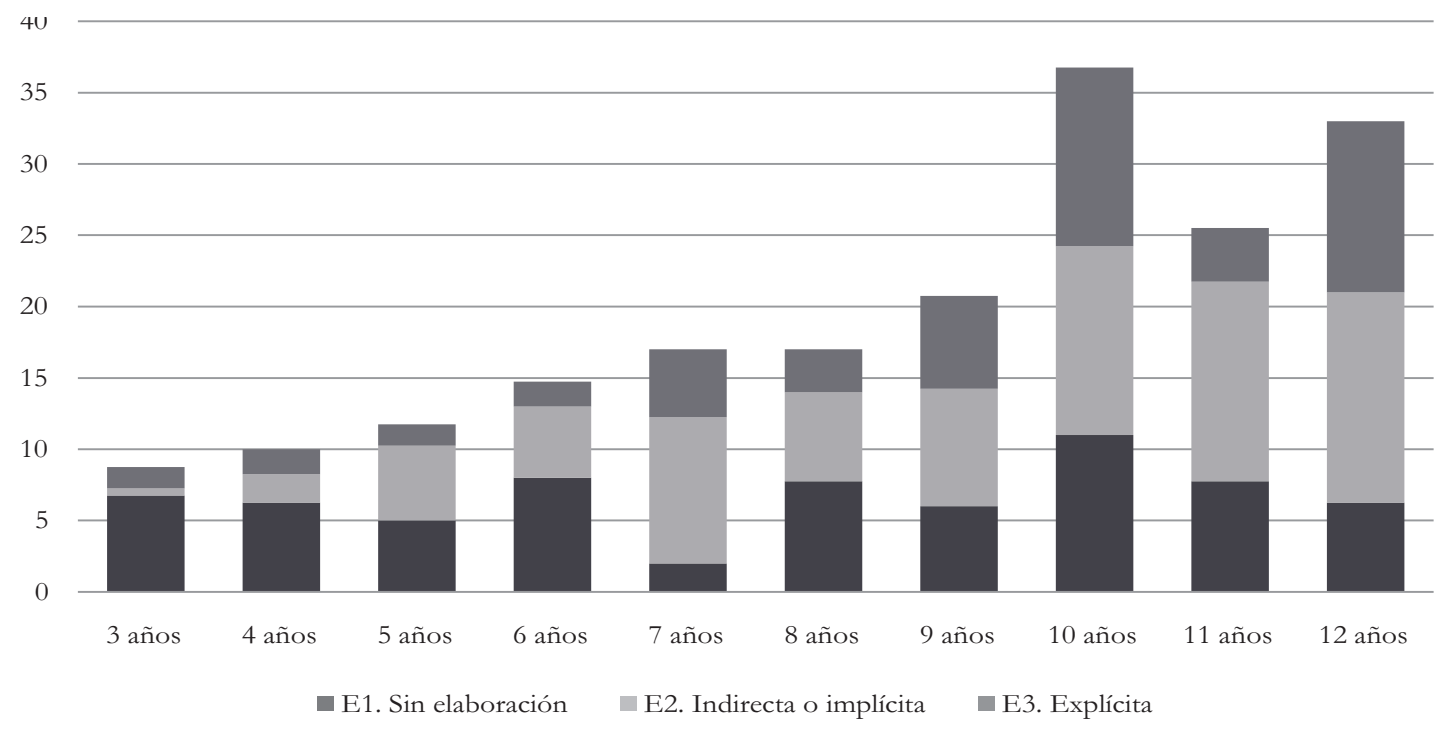

Figura 3. Distribución de las evaluaciones por nivel de elaboración. Medias por edad en el total de los reltatos $(N=40)$.

Los hallazgos hasta ahora reportados permiten observar diferencias relacionadas con la edad en las características y el nivel de elaboración de las evaluaciones hechas por los participantes en sus narraciones. Estas diferencias son más notorias a partir de los 7 y de los 10 años. Tanto la expresión de emociones como de comentarios muestran un cambio ascendente sensible a partir de los 10 años, en tanto que el nivel de elaboración es incipiente hasta los seis años, mostrando un avance notable a partir de los siete años. 
Tomando en cuenta estas diferencias, se agruparon los datos en los siguientes grupos de edad: 3 a 6 años, 7 a 9 años y 10 a 12 años; para realizar un análisis de varianza (ANOVA) que permitiría, por un lado, poner a prueba las hipótesis planteadas en la elaboración de las subcategorías y para determinar si las tendencias encontradas son estadísticamente significativas.

En la tabla 4 se presentan los datos descriptivos de las categorías, subcategorías y niveles de elaboración por grupo de edad. Los resultados muestran tendencias claramente ascendentes de todas las variables por grupo de edad, con excepción de los comentarios personales (CO_Personal) y el nivel de elaboración 1 (E1. Sin elaboración).

El análisis de varianza ANOVA de un factor (Grupo de edad) con post-hoc Bonferroni (ver tabla 5), mostró que hay diferencias significativas de Emociones, Comentarios, CO_General y E2. Indirecta o implícita entre todos los grupos; en EM_Estado, CO_Personal entre los grupos 2-3 y 1-3; y solo diferencias entre los más pequeños y los más grandes (Grupos 1 y 3) en EM_
Acción y E3. Explícita. Además, se analizaron las diferencias entre las variables por sexo, no encontrándose diferencias significativas entre niñas y niños.

En cuanto a las hipótesis, ya los datos descriptivos habían mostrado que las emociones de acción (EM_Acción) no son más frecuentes que las de estado (EM_Estado), contrariamente a lo anticipado. Esto se confirma con el análisis de varianza pues solo se encontraron diferencias significativas de evaluaciones de acción (EM_ Acción) entre los grupos extremos (3 a 6 años y 10 a 12 años) ya que de los 3 a los 9 años la producción es muy escasa y asciende ligeramente hacía los 10 años, en tanto que las evaluaciones de estado (EM_Estado) son frecuentes desde las edades tempranas.

Por otra parte, los datos descriptivos arrojan evidencia de que, como se anticipó, los comentarios generales (CO_General) aparecen de manera frecuente y desde edades más tempranas que los comentarios personales (CO_Personal). De hecho, el incremento

Tabla 4

Descriptivos por categoría, subcategoría y grupo de edad $(n=40)$

\begin{tabular}{lcccccc}
\hline & \multicolumn{3}{c}{3 a 6 años $(n=16)$} & 7 a 9 años $(n=12)$ & \multicolumn{1}{c}{10 a 12 años $(n=12)$} \\
\cline { 2 - 7 } \multicolumn{1}{c}{ Tipo de Evaluación } & Media & DE & Media & DE & Media & DE \\
\hline Emociones & 7.81 & 3.83 & 11.25 & 2.56 & 16.00 & 3.64 \\
/EM_Acción & 1.75 & 1.48 & 2.67 & 0.49 & 3.42 & 1.44 \\
/EM_Estado & 6.06 & 2.89 & 8.58 & 2.5 & 12.58 & 3.12 \\
Comentarios & 3.5 & 3.1 & 7.00 & 2.95 & 15.75 & 3.93 \\
/CO_General & 3.5 & 3.1 & 7.00 & 2.95 & 14.42 & 3.75 \\
/CO_Personal & 0 & 0 & 0 & 0 & 1.33 & 2.31 \\
Nivel de Elaboración & & & & & & \\
/E1. Sin elaboración & 6.5 & 4.62 & 5.25 & 3.25 & 8.33 & 5.12 \\
$\quad$ /E2. Indirecta o & 3.19 & 2.64 & 8.25 & 3.39 & 14.00 & 4.43 \\
implícita & & & & & & \\
/E3. Explícita & 1.63 & 1.71 & 4.75 & 3.02 & 9.42 & 8.22 \\
\hline
\end{tabular}


Tabla 5

Resultados ANOVA categorías y subcategorías por grupos de edad y post-hoc Bonferroni

\begin{tabular}{|c|c|c|c|c|}
\hline \multirow[b]{2}{*}{ Categorías/Subcategorías } & \multirow{2}{*}{$\begin{array}{c}\text { ANOVA } \\
F \\
g l=2,37\end{array}$} & \multicolumn{3}{|c|}{ Bonferroni - Diferencias significativas $\sqrt{ }$} \\
\hline & & $\begin{array}{c}\text { Gpos. 1-2 } \\
3 \text { a } 6 \text { años vs. } \\
7 \text { a } 9 \text { años }\end{array}$ & $\begin{array}{c}\text { Gpos. 2-3 } \\
7 \text { a } 9 \text { años vs. } \\
10 \text { a } 12 \text { años } \\
\end{array}$ & $\begin{array}{c}\text { Gpos. 1-3 } \\
3 \text { a } 6 \text { años vs. } \\
10 \text { a } 12 \text { años } \\
\end{array}$ \\
\hline Emociones & $(19.387, p<.001)$ & $\sqrt{ }$ & $\sqrt{ }$ & $\sqrt{ }$ \\
\hline EM_Estado & $(17.973, p<.001)$ & & $\sqrt{ }$ & $\sqrt{ }$ \\
\hline EM_Acción & $(6.124, p<.005)$ & & & $\sqrt{ }$ \\
\hline Comentarios & $(47.562, p<.001)$ & $\sqrt{ }$ & $\sqrt{ }$ & $\sqrt{ }$ \\
\hline CO_General & $(38.828, p<.001)$ & $\sqrt{ }$ & $\sqrt{ }$ & $\sqrt{ }$ \\
\hline CO_Personal & $(7.467, p<.015)$ & & $\sqrt{ }$ & $\sqrt{ }$ \\
\hline \multicolumn{5}{|l|}{ Elaboración } \\
\hline E2. Indirecta o implícita & $(33.266, p<.001)$ & $\sqrt{ }$ & $\sqrt{ }$ & $\sqrt{ }$ \\
\hline E3. Explícita & $(8.692, p<.001)$ & & & $\sqrt{ }$ \\
\hline
\end{tabular}

de comentarios generales (CO_General) es significativo entre los tres grupos de edad. En cuanto al nivel de elaboración, este se va haciendo más complejo con la edad, como se había anticipado; la frecuencia elaboración tipo 2 (E2. Indirecta o implícita) muestra diferencias significativas en los tres grupos y la tipo 3 (E3. Explícita) entre el primero y el tercer grupo.

Estos resultados permiten plantear que la expresión de evaluaciones en los participantes de esta submuestra $(n=40)$ presenta una progresión por tipo y nivel de elaboración que puede describirse en tres etapas.

Etapa I. Entre los tres y los seis años, las narraciones incluyen principalmente evaluaciones de emoción de estado (EM_Estado), del tipo: feliz, triste, enojado, alegre, contento, y algunos comentarios generales (CO_Generales); rara vez estas evaluaciones se relacionan con los sucesos que se relatan, es decir, aparecen más bien como expresiones aisladas (E1.Sin elaboración) por lo que es difícil, a partir del discurso, identificar su causa o su consecuencia, lo que hace que la comprensión de las evaluaciones dependa de la atención a la secuencia de imágenes por parte del oyente.

En los ejemplos siguientes, se ofrece una sinopsis del contenido de las láminas según el relato del que se trata. En la transcripción de la narración se subrayan los segmentos que incluyen la evaluación y se indica en cursivas el código del tipo de evaluación con el nivel de elaboración en paréntesis.

Ejemplos de relatos típicos de la Etapa I:

Relato 6. Una rana se queda sola en un parque y se come un insecto, descubriendo que no es lo que creía. (FEM 4 años. exp. 018)

Unas mariposas estaban volando y una niña iba con su perrito a caminar. Y una rata, una rana la encontró.

La mariposa se estaba feliz. EM_Estado(1)

Y una rata, la rana quería comer a la abeja. $C O_{-}$ General(1) 
"Guácala, no me la (inaudible) tener que comer", dijo: "voy a chupar, voy a comerme esto", dijo: "¿a poco?". CO_General(1)

Relato 4. Una rana se escapa del frasco donde la tiene un niño y éste la busca en el bosque. (MASC 6 años, exp. 040)

El perro y su ama. Un perro y la rana. Y ahí dejó el niño sus botas. Y la rana se está metiendo a una botella.

Y luego el siguiente día, este, se queda el niño: “oh!". Y el perro encima, arriba del niño y luego dice, CO_General(1)

Y luego el perro le pega al panal. Y luego el niño: “oh! Cielos!". Y el niño las, y el perro las trata de atrapar. CO_General(1)

Y luego querían atrapar una cosa. Y se metieron a un tubo.

Y luego y ya, y estaban viendo la rana y con otra rana, pero era una mujer y un hombre y sus hijitos. Y luego ahí estaban con sus hijos.

Y luego el niño les estaba lanzando cosas y "paz" no las lanzó. CO_General(1)

Y el perro estaba muy feliz. EM_Estado(1)

Y él, y la rana de su niño se había aven, se cayó al agua. Ya.CO_General(1)

Etapa II. Entre los siete y los nueve años, las evaluaciones consisten en el mismo tipo de expresiones que en la etapa anterior, emociones de estado (EM_Estado) y comentarios generales (CO_Generales), pero se observa un avance en la elaboración del discurso, pues el narrador conecta, por yuxtaposición o mediante nexos copulativos, los antecedentes o las consecuencias de sus expresiones evaluativas (E2. Implícita o indirecta), ocasionando una menor dependencia en la imagen por parte del oyente.

Ejemplos de relatos típicos de la Etapa II:

Relato 7. Un niño recibe de regalo una ranita, por lo que su perro y su tortuga se ponen felices, pero su rana se pone celosa. (FEM 7 años, exp. 050)
Había una vez un niño que era Navidad y trajeron su regalo. Tenía de mascota un perro, una tortuga y una rana. CO_General(2)

Lo abrió y era una rana y la otra rana se puso furiosa y los demás felices. EM_Estado(2)

Luego los presentó y los demás seguían felices. EM_Estado(2)

Y luego la rana mordió a la que habían traído.

Y luego ellos se pusieron muy tristes. EM_ Estado(2)

Y el niño se quedó admirándolo (admirado). EM_ Estado(2)

Y luego el niño estaba diciendo: "no". $\mathrm{CO}_{-}$ General(2)

Y los demás se estaban furiosos y eso porque había venido una rana nueva. EM_Estado(3)

Relato 7. Un niño recibe de regalo una ranita, por lo que su perro y su tortuga se ponen felices, pero su rana se pone celosa. (FEM 9 años, exp. 068)

Era de un niño que le mandaron un regalo. $\underline{Y}$ estaba sorprendido, con sus tres amigos. EM_ Estado(2)

El niño abrió el regalo y vio que era una rana $\underline{\underline{ }}$ todos estaban sorprendidos menos la otra rana que tenía el niño. EM_Estado(2)

El niño trató de presentarle a la otra rana que estaba un poco celosa porque estaba la rana. $\mathrm{CO}_{-}$ General(3)

Luego la rana mordió la otra rana que le habían regalado al niño.

Luego el niño agarró la otra rana que le regalaron y le dijo que no lo hubiera, hubiera hecho eso la otra rana. Y ya.

Etapa III: A partir de los 10 años, los niños y las niñas ofrecen una mayor cantidad de evaluaciones y comienzan a utilizar más recursos discursivos para hacer explícita la relación que esa evaluación tiene con los sucesos. Así, comienzan a poner en práctica, unos más que otros, recursos como la explicación o la justificación a través de nexos 
causales e ilativos, e incluso frases comparativas (ej. “de tanto que...”), apoyando con ello la comprensión del oyente aún con poca atención a la imagen (E3. Explícita) y comienzan a ofrecer algunos comentarios personales (CO_Personal) que muestran su posicionamiento frente a los sucesos narrados.

Ejemplos de relatos típicos de la Etapa III:

Relato 1. Una pareja está de día de campo y una rana los interrumpe. (FEM, 10 años, exp. 071)

Bueno, esta son dos esposos y fueron de picnic porque era un bonito día, $C O \_G e n e r a l(3)$

Entonces la rana que apareció en el otro cuento, los vio corriendo CO_General(2)

y va y vio una rica canasta con sándwich, sí eran sándwich. CO_General(2)

Entonces aprovechó a los esposos cuando estaban distraídos, la ranita aprovechó para meterse y agarrar un sándwich y comérselo. CO_General(2)

Entonces cuando la esposa quería sacar un sándwich, la ranita se aferró a su mano pensando que era un sándwich.EM_Estado(3)

Cuando sacó la mano vio una ranita y se asustó mucho.EM_Estado(3)

Entonces muy enojada porque ya no iba a comer su sándwich/porque de seguro estaba todo chupado y baboso, entonces aventó a la ranita y la agarró y lo aventó. CO_General(3)

Y la ranita se fue feliz porque ya había comido. EM_Estado(3)

Relato 7. Un niño recibe de regalo una ranita, por lo que su perro y su tortuga se ponen felices, pero su rana se pone celosa. (MASC 12 años, exp. 091)

Estaban, un niño, un, su perro, una rana y la tortuga. Yo creo que era su cumpleaños, porque tenía un, una caja de regalo enfrente. CO_Personal(3)

Entonces lo destapa y había den, y lo que estaba adentro era una rana.

El perro y la tortuga estaban muy felices, $E M_{-}$ Estado(2)
Pero la otra rana estaba muy enojada/porque creyó que la iban a EM_Estado(3)

remplazar por la que estaba en la caja. $\mathrm{CO}_{-}$ General(3)

Entonces estaban todos felices y le presentan la rana que estaba en EM_Estado(2)

la caja a la rana que ya tenía más tiempo, ahí viviendo con el niño. CO_General(3)

Entonces se enoja y le muerde la patita. EM_ Estado(2)

Entonces el niño se asusta. EM_Estado(2)

Los perros, el perro y la tortuga se sorprenden. EM_Estado(2)

$\underline{Y}$ al final re, los, los cuatro regañan a la rana. $E M_{-}$ Acción(2)

\section{Discusión}

Este trabajo se realizó con base en narraciones producidas a partir de láminas de los libros de la colección de "Frog" en niños de 3 a 12 años. Se retomaron datos de una investigación previa y se reanalizaron los correspondientes a la categoría de "evaluación" para identificar subtipos de "emociones" y de "comentarios", además se clasificaron las emisiones evaluativas en tres niveles de elaboración discursiva.

Los resultados muestran una progresión que involucra tanto la cantidad como la calidad de expresiones evaluativas. Estos hallazgos coinciden con lo encontrado por otros investigadores que han trabajado con población hispana y no hispana, así como con distintas metodologías de producción narrativa (Bamberg \& Reilly, 1996; Fernández \& Melzi, 2008; Jiménez Van Der Biest, 2006; Shiro, 2008).

En general, se observa un incremento de la cantidad y calidad en la evaluación narrativa a partir de los siete años. Esto podría explicarse por la mayor capacidad de los niños para 
comprender emociones, tal y como lo demuestran Aldrich et al. (2011), quienes además de obtener narraciones con un libro sin texto (utilizado también en esta investigación), aplicaron una prueba de comprensión de emociones.

La escasa presencia de evaluaciones antes de los siete años coincide con lo hallado por investigadores que han trabajado con población anglosajona (Bamberg \& Reilly, 1996). Otros autores reportan incrementos alrededor de los 10 años ( $5^{\circ}$ grado), que coinciden con la tercera etapa identificada en este estudio (Jiménez Van Der Biest, 2006). La revisión de estudios realizada por Bamberg y Reilly, además, coincide en gran medida en cuanto a la descripción cuantitativa y cualitativa de las narraciones que hemos identificado en este trabajo.

A pesar de que dos subcategorías tuvieron muy poca productividad, "comentarios personales" y "emociones de acción", estos hallazgos son congruentes con la literatura. Por ejemplo, Shiro (1998) plantea que los niños inicialmente expresan sus evaluaciones en tercera persona $y$ posteriormente en primera persona pero incluso a los 10 años, algunos autores como Jimenez Van Der Biest (2006), reportan una baja incidencia de este rasgo. La presencia de comentarios personales, una de cuyas características es el uso de la primera persona, fue escasa; sin embargo, ésta mostró un repunte en el extremo superior, los 12 años. Además, existen indicios en poblaciones hispanas de que las evaluaciones en primera persona son más frecuentes en las narraciones personales que en las de ficción (Shiro, 2008).

La subcategoría de "emociones de acción" no parece representar un desafío en términos de desarrollo, los niños y niñas expresaron pocas emociones de este tipo (llorar, gritar, etc.) a lo largo de todas las edades, lo que sugiere que su ocurrencia está más bien asociada con el material y no con las habilidades de los narradores. La influencia del material, y por tanto del tipo de narración, ha sido reportada como una variable relevante. Por ejemplo, Fernández y Melzi (2008) reportan una mayor presencia de evaluaciones sobre estados internos, categoría similar a las "emociones de estado" de este trabajo, en narraciones de ficción que en narraciones personales.

Este estudio confirma algunos hallazgos previos en relación con la evolución de la evaluación narrativa y ofrece información adicional y específica de niños hispanohablantes que nos permite avanzar en nuestra comprensión de cómo las niñas y los niños van construyendo este elemento esencial del género narrativo.

La propuesta de etapas derivada de este trabajo, puede ser de utilidad para maestros de educación básica para valorar las narraciones de ficción de sus alumnos, así como para planificar intervenciones que apoyen su desarrollo narrativo, tanto oral como escrito.

Algunas de las limitaciones de este trabajo como el tamaño de la muestra, la ausencia de un grupo de comparación por nivel socioeconómico, la falta de estímulos de contraste para obtener narraciones personales, principalmente, constituyen una agenda de investigación futura para profundizar en el conocimiento de la narración infantil.

\section{Referencias}

Aldrich, N., Tenenbaum, H., Brooks, P., Harrison, K., \& Sines, J. (2011). Perspective taking in children's narratives about jealousy. British Journal of Developmental Psychology, 29, 86-109. 
Bamberg, M. \& Reilly, J. (1996). Emotion, narrative, and affect: How children discover the relationship between what to say and how to say it. En D. Slobin, J. Gerhardt, A. Kyratzis \& J. Guo (Eds.), Social Interaction, Social Context and Language: Essays in Honor of Susan Erwin-Tripp (pp. 329-341). Hillsdale, NJ: Lawrence Erlbaum.

Fernández, C. \& Melzi, G. (2008). Evaluation in Spanish-speaking mother-child narratives: The social and sense-making fuction of internal-state references. En A. McCabe, A. L. Bailey \& G. Melzi (Eds.), Spanish-language narration and literacy. Culture, cognition and emotion (pp. 92-118). Cambridge, MA: Cambridge University Press.

Gómez Martínez, G. E. (2012). Diseño y piloteo de la Prueba de Evaluación Narrativa (PEN) para niños de tres a doce años. San Luis Potosí, México: Tesis de maestría no publicada. Universidad Autónoma de San Luis Potosí.

Hess, K. (2010). Saber lengua. Lenguaje y metalenguaje en los años escolares. México, D.F.: El Colegio de México.

Instituto Nacional para la Evaluación de la Educación. (2007). La educación para poblaciones en contextos vulnerables. Mexico, D. F.: INEE.

Jiménez Van Der Biest, T. (2006). La narración infantil. Un estudio en niños de educación básica. Revista de Investigación, 60, 157-174.

Labov, W. \& Waletzky, J. (1967). Narrative analysis: Oral versions of personal experience. Journal of narrative and life historiy, 7 (1-4), 3-38.
Levey, S. (2003). Reported dialogue and pragmatic particles in the narratives of preadolescents. World Englishes, 22 (3), 305-321.

Mayer, M. (1967). A boy, a dog and a frog. New York: Dial Books for Young Readers.

Mayer, M. (1969). Frog, where are you? New York: Dial Books for Young Readers.

Mayer, M. (1973). Frog on his own. New York: Dial Books for Young Readers.

Mayer, M. \& Mayer, M. (1971). A boy, a dog, a frog and friend. New York: Dial Books for Young Readers.

Mayer, M. \& Mayer, M. (1975). One frog, too many. New York: Dial Books for Young Readers.

Shiro, M. (1998). Los pequeños cuentacuentos. El desarrollo de las habilidades narrativas de niños en edad escolar. Trabajo de ascenso inédito. Caracas: Universidad Central de Venezuela.

Shiro, M. (2003). Genre and evaluation in narrative development. Journal of Child Language, 30, 165195.

Shiro, M. (2008). Narrative stance in Venezuelan children's stories. En A. McCabe, A. L. Bailey \& G. Melzi (Eds.), Spanish-language narration and literacy. Culture, cognition and emotion (pp. 213-236). Cambridge, MA: Cambridge University Press.

Stein, N., \& Albro, E. (1997). Building complexity and coherence: Children's use of goal-structured knowledg in telling stories. En M. Bamberg (Ed.), Narrative development: Six approaches (pp. 5-44). Mahwah, NJ: Lawrence Erlbaum

Recibido: 04 de abril de 2013

Aceptado: 21 de agosto de 2013 